\title{
Chapter 4 \\ Indirect Contact Interventions to Promote Peace in Multicultural Societies
}

\author{
Reeshma Haji and Hiromi Noguchi
}

\subsection{Introduction}

As societies become more diverse through immigration and refugee settlement, promoting more favourable attitudes between groups of different cultural backgrounds is of pressing importance. An unprecedented number of 68.5 million forcibly displaced people, with more than 28 million refugees, have been reported by the UNHCR (2018). Reactions to newcomers are mixed: Western Europeans are more favourable toward a decrease in immigration than an increase (Pew Research Centre, 2018), and immigrants from North Africa and the Middle East are viewed especially negatively (Pew Research Centre, 2018). Research shows that young children learn prejudice through adults' instruction and these "social rules" shape children's understanding of their social worlds (Kang \& Inzlicht, 2012). Now more than ever, it seems that an attitude of acceptance of the other, acceptance, and even the celebration of difference need to be instilled from a young age. This chapter describes some methods for fostering these favourable views of the other.

We situate this chapter within the broader context of peace psychology. Interventions aimed at promoting more favourable views of the other may help to reduce structural violence. This violence is inherent in social systems and has its effects by exposing people to inequality and injustice (Christie, Wagner, \& Winter, 2001). Seeing the other as equal could enable the challenging of legitimizing myths that maintain unequal social structures. We describe interventions to be used with

Thank you to Cailynn Laprise, Rachel Vandervecht, and Burgandy Thiessen for research assistance.

R. Haji $(\bowtie) \cdot$ H. Noguchi

Laurentian University, Sudbury, ON, Canada

e-mail: rhaji@laurentian.ca 
children and youth, for those who endeavour to prepare young people to confront structural violence over their lifetimes. The interventions are grounded in social psychology, specifically a key theoretical perspective known as intergroup contact.

Intergroup Contact Theory, articulated by Allport (1954), posited that bringing groups into physical contact with each other would promote more positive attitudes toward the other. This ought to be particularly the case when the interaction is marked by optimal conditions, including equal status, common goals, cooperation between the groups, and the support of authority or societal customs. This theory inspired decades of research in various parts of the globe. A very influential metaanalysis (statistical integration) of 515 studies found that, overall, physical interaction of groups decreases prejudice (Pettigrew \& Tropp, 2006). Indeed, intergroup contact can promote more favourable intergroup attitudes even when optimal conditions are absent. Moreover, in a process called secondary transfer, positive attitudes developed through exposure to one group can spread to other groups not involved in the interaction (Pettigrew, 2009). For example, research with Canadian undergraduate students found that having someone from a different religious group among one's five closest friends is associated with greater openness toward other religious groups beyond the friends' (Haji \& Lalonde, 2017).

Peace practitioners and scholars alike recognize bringing different groups into direct interaction with each other is not always easy. First, people may live in homogeneous societies or segregated settings. Second, the groups may be unwilling to interact with each other. Third, bringing groups together for an in-person interaction may be costly or impractical. Fourth, particularly in conflict or post-conflict societies, there is the potential for a direct interaction to go badly or for people to have anxiety about interaction. Therefore, recent research has focused on the use of indirect contact as a means of promoting positive attitudes between groups. This chapter provides (1) a description of these methods and how they have been supported by research with children and youth, (2) suggestions for optimizing these methods for practical application, and (3) future directions for research and interventions in this area.

\subsection{Methods of Indirect Intergroup Contact}

This section describes methods of indirect intergroup contact and evidence of their effectiveness based primarily on research with children and youth. Indirect contact is an umbrella term referring to methods of intergroup contact in which the groups do not come into direct physical interaction with each other. Three methods will be described, including extended contact, vicarious contact, and imagined contact. 


\subsubsection{Extended Contact}

Extended contact (Wright, Aron, McLaughlin-Volpe, \& Ropp, 1997) refers to the knowledge that someone from one's own group, the ingroup, has a close relationship, such as a friendship, with someone from an outgroup, or another group to which one does not belong. Initial research on this form of indirect contact (Wright et al., 1997) found that White American undergraduate students with close friends who had friends from other ethnic groups reported having more favourable attitudes toward those ethnic groups, even when their own friendships with other ethnic groups were controlled for statistically. This correlational research provided evidence for extended contact benefits being associated with participants' knowledge of their real pre-existing friendships, but no causal conclusions could be drawn. Therefore, the researchers also experimentally investigated the effects of extended contact on outgroup attitudes. One of these studies used an established method of creating conflict and rivalry between two groups in a laboratory setting. The groups were then separated and one member from each group was paired together. The pair engaged in a closeness-building task dubbed "the fast friends procedure", gradually revealing more information about themselves by taking turns discussing topics provided by the researchers. Then, each member of the pair was reunited with their original group and was asked to inform their group members of their experience with the member of the other group. Participants' reactions were measured on outcomes such as intergroup discrimination and perceived quality of intergroup relations at various points in the study. This experiment found that extended contact, in the form of learning about one's ingroup member having close contact with an outgroup member, led to decreased discrimination (favouring one's own group over the other group) and improved perceptions of the quality of intergroup relations.

Much of the research on extended contact has been carried out with children in Europe. For example, research by Lindsey Cameron and her colleagues in the UK shows that extended contact through story reading can promote more favourable outgroup attitudes. In one study (Cameron \& Rutland, 2006), British schoolchildren aged 5-10 took part in a 6-week program that involved reading stories with them about children similar to themselves who had close friendships with a stigmatized outgroup (disabled children). All characters in the stories were presented favourably. After the story reading, there were small group discussions. Participants were encouraged to remember the story content and it was emphasized that the characters were typical members of their groups. These story-related activities occurred once a week for a period of 6 weeks. Participants were interviewed 1 week prior to and 1 week following the intervention, to see whether there were changes in attitudes and intended behaviour toward the outgroup. The results suggested that extended contact through story reading did indeed improve non-disabled children's attitudes and intended behaviour toward disabled children. Extended contact was most effective when the group categories (disabled and non-disabled) and the typicality of the characters (as average members of their groups) were emphasized. 
Similar extended contact interventions with storybooks have been used to improve children's attitudes toward refugee children (Cameron \& Rutland, 2007, Study 2; Cameron, Rutland, Brown, \& Douch, 2006). For example, Cameron et al. (2006) tested the effectiveness of different variations of extended contact on British children's attitudes toward refugees. Schoolchildren belonging to two different age groups (5-8 years and 9-11 years) were exposed to a 6-week intervention involving reading and discussing stories involving friendships between British children and refugee children. Participants were exposed to one of three types of extended contact stories: (1) stories that focused on the individual characteristics of the British and refugee children; (2) stories that focused on the fact that the British and refugee children belonged to a common group, i.e. students at the same school; or (3) stories that focused on the group memberships (British and refugee) but also emphasized that the students belonged to a common group. Participants in the third group (called the dual identity group) reported the most favourable reactions toward refugee children. The study also found that this intervention was more effective for younger children than older children.

Other research has focused on existing popular books, rather than stories that have been specially designed for extended contact interventions. For example, in research conducted with elementary and secondary schoolchildren in Italy and undergraduate students in the UK (Vezzali, Stathi, Giovanni, Capozza, \& Trifiletti, 2015), it was found that reading Harry Potter books improved participants' attitudes toward a variety of outgroups, such as immigrants, homosexuals, and refugees. Harry Potter books were selected because the main character, Harry, befriends characters who belong to various stigmatized groups. The researchers argued that fantasy books, such as Harry Potter, in which group memberships are fictional, offer greater potential of generalization of positive attitudes from extended contact to a variety of real-world stigmatized groups.

As another example, Vezzali, Stathi, and Giovanni (2012) conducted a study with Italian adolescents (with an average age of 12), who were given a summer reading list consisting of intercultural books (dealing with positive interaction between characters of different cultures, including a character from a culture similar to that of participants), non-intercultural books, or no summer reading list. One week after the start of the following school year, participants' reactions toward immigrants were assessed. Importantly, this research assessed not only attitudes toward immigrants, but behavioural intentions and specifically the desire to engage in direct contact with immigrants. Results indicated that students in the intercultural reading group reported more favourable views of immigrants, greater closeness to them, and a greater desire for future contact with them.

Extended contact research has also been used in segregated settings where there is a clear history of conflict, such as on the divided island of Cyprus (Husnu, Mertan, \& Cicek, 2018). Intergroup violence erupted between Turkish and Greek communities in the 1970s and the result was a divided island with Turkish Cypriots residing in the North and Greek Cypriots in the South. This is a context where researchers have noted the importance of intervention at a young age, before children are exposed to considerable negative information about "the other" in stories, legends, 
historical accounts, and more. In one study (Husnu et al., 2018; Study 1), Turkish Cypriot children (aged 6 to 12) were assessed for both positive and negative extended contact in the form of stories heard in their families about Greek Cypriots. The researchers found that positive family storytelling about Greek Cypriots predicted children's more favourable attitudes toward them, even after the positive effects of direct contact had been taken into account.

Recently, Vezzali, Hewstone, Capozza, Giovannini, and Wöelfer (2014) published a review of the scholarly literature on extended contact, as well as vicarious contact (discussed in the next section), and found that many of those studies statistically controlled for direct contact. Therefore, these forms of indirect contact were associated with more favourable responses toward the outgroup, even when direct contact had been taken into account. Moreover, indirect contact effects were stronger among those with limited direct contact experience. Additionally, indirect contact was associated with more favourable outgroup attitudes in different age groups and even in post-conflict settings. This review also noted that indirect contact effects have been observed on diverse outcomes such as thoughts, feelings, and behaviours toward the other group.

\subsubsection{Vicarious Contact}

Vicarious contact refers to the observation of someone from the ingroup interacting with someone from the outgroup. It makes use of the social learning perspective and involves an ingroup member modelling favourable reactions toward the outgroup (Mazziotta, Mummendey, \& Wright, 2011; Wright et al., 1997). Participants may observe in-person interactions or do so via some medium, such as a video or storybook.

Vicarious contact has also been used with children in post-conflict settings. In another study in Cyprus (Husnu et al., 2018; Study 2), Turkish Cypriot children (aged 6 to 11) were involved in a 3-week intervention that consisted of 30-minute sessions of having stories read to them about Turkish Cypriot children who had friendships with Greek Cypriot children. Stories were followed by small group discussions and the typicality of the characters was emphasized. This intervention improved Turkish Cypriot children's attitudes, trust, and intended behaviour toward Greek Cypriot children.

In some interventions, including the storybook methods described above, extended contact and vicarious contact can occur in parallel. The core idea of extended contact is the knowledge that an ingroup member has a positive relationship with an outgroup member (Wright et al., 1997). Vicarious contact goes a step beyond mere knowledge, involves the observational learning from an ingroup member interacting with an outgroup member, and can in some instances co-occur with extended contact. For example, anxiety may be reduced via observation of a cross-group friendship that may be afforded through extended contact. Additionally, observation of a friendly interaction between an ingroup and outgroup member 
provides information (knowledge) that can change negative outgroup stereotypes. Importantly, however, extended contact can occur without vicarious contact as a person may be aware of a friend's friendship with an outgroup member without having had the opportunity to observe an interaction between them.

\subsubsection{Parasocial Contact}

One subtype of vicarious contact is exposure to an outgroup member through mass media, such as television or radio, and this has sometimes been referred to as parasocial contact. Researchers have investigated the effects of vicarious contact through video on attitudes toward the outgroup and willingness to engage in future interactions with them (Mazziotta et al., 2011). In one study, German young adults (aged 18 to 28 ) were exposed to 2 video clips that showed friendly interactions between a German student and a Chinese student (parasocial contact group) or 2 video clips that showed friendly interaction between two German students (control group). Both groups also saw a neutral video clip about campus library facilities. The parasocial contact group reported more favourable attitudes and feelings about Chinese people, greater willingness to interact with them, more positive future expectations, and less uncertainty about such interactions. In a follow-up study, the researchers demonstrated that their effects were not due to mere exposure to the outgroup. Only video exposure to intergroup contact with a Chinese student, and not mere video exposure to a Chinese student, produced the more favourable reactions toward Chinese people.

In this research, it was also demonstrated that the parasocial contact made use of two different forms of social learning: (1) Observational knowledge about how to behave appropriately with the outgroup and (2) Positive expectations regarding future interactions with the outgroup (self-efficacy expectancy). Given that interaction with a different group can be anxiety-provoking, optimistic expectations regarding direct contact with the outgroup could be learned through parasocial contact. They suggested that this was particularly the case when the role models that were being observed were similar to participants being exposed to the intervention.

\subsubsection{Parasocial Exposure Without Cross-Group Interaction}

Intervention studies with youth have found that vicarious contact with a positive intergroup interaction may be essential to improving outgroup attitudes in a parasocial intervention. One study investigated the effects of parasocial contact with two 13-yearold gay male characters on LGBTQ attitudes among youth aged 13 to 21 (Gillig \& Murphy, 2016). Participants were exposed to a 9-minute video about the budding romance between the characters or did not view the video and then completed an attitude questionnaire. Whereas the parasocial exposure to gay youth improved 
LGBTQ attitudes among LGBTQ youth, it actually worsened attitudes among heterosexual youth. Importantly, observation of a cross-group friendly interaction, rather than parasocial exposure to the outgroup, may be the essence of vicarious contact intervention.

\subsubsection{Imagined Contact}

Imagined contact refers to the mental simulation of an interaction with an outgroup member (Turner, Crisp, \& Lambert, 2007). Some initial research in this area involved undergraduate students in the UK. In one study, heterosexual male participants aged 19 to 25 imagined engaging in a conversation with a gay man that they had met on a train or spent the same amount of time imagining a nature hike (Turner et al., 2007; Experiment 3). Results indicated that those who imagined the interaction with the gay man had more positive attitudes toward homosexuals than those who imagined the nature hike. Subsequent research demonstrated that in order to optimize effects of imagined contact intervention, an imagined interaction needs to be positive and mental simulation of the event should be encouraged (Crisp, Stathi, Turner, \& Husnu, 2009). Additionally, a practical teaching and learning guide related to imagined contact was provided by Stathi and Crisp (2009).

There is evidence of imagined contact's effects on young people's willingness to interact with "the other". For example, research from the UK (Turner, West, \& Christie, 2013) found that high school students who imagined an interaction with an asylum seeker reported greater intentions to interact with asylum seekers. Another study by the same authors (Turner et al., 2013) also found that undergraduate students who imagined an interaction with a gay person reported a stronger intention to interact with gay people and greater trust and less anxiety about interacting with the other group. Other research in the UK context involved a 3-week intervention with schoolchildren (Stathi, Cameron, Hartley, \& Bradford, 2014). White elementary schoolchildren who imagined an interaction with an Asian child on a weekly basis were compared to another group who did not engage in this activity over the same period. Again, imagined contact resulted in greater willingness to interact with the other group, as well as greater perceived similarity to them.

A meta-analysis of 71 studies on imagined contact (Miles \& Crisp, 2014) confirmed that imagined contact can promote more favourable responses toward an outgroup and elaboration is an important factor in imagined contact - the more detail in which participants imagined the interaction, the greater the positive effects of imagined contact. A striking finding of the meta-analysis was that although imagined contact has been effective in promoting more favourable intergroup attitudes in adults and in children, effects of these interventions are strongest with children. This may be due to the importance of imagery in children's day-to-day lives and their potentially greater facility with imagination tasks.

Furthermore, this meta-analysis confirmed that imagined contact improved attitudes, emotions, intended behaviour, and actual behaviour toward the outgroup. 
Interestingly, the effect on intended behaviour was greater than the effect on attitudes. Although the effect on actual behaviour was similar to that on intended behaviour, fewer studies existed on actual behaviour. Therefore, imagined contact has the potential for improving intended and actual behaviour toward other groups, not just improving attitudes. This is encouraging, as it's often behaviour that we are actually trying to change.

Imagined contact can be used as practice or prior preparation for actual contact as research shows that imagined contact reduces anxiety about interacting with the other group. Use of imagination for anxiety reduction has long been used in clinical settings (e.g., for treating phobias), and imagination can also be used outside the clinic for intervening prejudice toward those of other cultural backgrounds (Birtel \& Crisp, 2012).

\subsection{Optimizing Methods for Practical Application}

As described above, there is considerable evidence for the effectiveness of indirect contact in improving attitudes toward the other. Indeed, research on real-world interventions found that there was no significant difference in effect sizes of direct and indirect contact interventions (Lemmer \& Wagner, 2015), though imagined contact was not included in this analysis. Meta-analyses suggest the effect size observed is a modest medium for real-word indirect contact interventions $\left(\mu_{\theta}=0.28\right.$; Lemmer \& Wagner, 2015) and for imagined contact $(d+=0.35$; Miles \& Crisp, 2014). There are various factors that should be considered to optimize these effects.

It is important to note that indirect contact interventions are particularly effective among those who have had little previous contact with the outgroup. For example, in our research (Haji \& Noguchi, 2017), we found that imagined contact promoted more favourable attitudes toward East Asians among those who did not have an East Asian person among their five closest friends. Similarly, in other research (Haji, McKeown, \& Stathi, 2017) that evaluated another variation of imagined contact (known as imagined contact with I-sharing, which is detailed below), we found that imagined contact with a Muslim person promoted more favourable attitudes toward Muslims among those who had not had previous contact with Muslims. Indeed, the greater effectiveness of indirect contact among those with limited direct contact experience has been noted in reviews of the literature (e.g. Vezzali et al., 2014). In a related vein, meta-analytic research has found that indirect contact is more effective in promoting favourable outgroup attitudes among majority group members, rather than among minority group members (Lemmer \& Wagner, 2015). It has been suggested that one reason for this is that minority group members have more contact experience with the majority, rather than vice versa.

Regardless of methods of contact (i.e. imagined, vicarious, or parasocial), exposing children and youth to cross-group friendship (positive interaction of ingroup members and outgroup members) is important for enhancing effectiveness 
of indirect contact interventions (e.g. Mazziotta et al., 2011; Miles \& Crisp, 2014; Vezzali et al., 2014).

For extended and vicarious contact, emphasizing group memberships, or making them salient, is important to augment the influence of these forms of indirect contact on outgroup attitudes (Cameron \& Rutland, 2006; Vezzali et al., 2014; Wright et al., 1997).

One theme that appears to be pervasive with indirect contact interventions with children is repeated exposure. Imagined contact and vicarious contact interventions described above typically took place over a period of 3 weeks (e.g. Husnu et al., 2018; Turner et al., 2013) and one of the extended contact interventions took place over a period of 6 weeks (Cameron \& Rutland, 2006). Whereas laboratory studies have detected indirect contact effects in a single session, it is likely the case that repeated exposure to indirect contact produces stronger or more enduring effects on attitudes and behaviour toward the outgroup. Indeed, researchers have argued that multiple sessions over a period of time may be required for lasting attitude change (e.g. Cameron \& Rutland, 2006). Based on past work, our recommendation is that interventions with children take place in multiple sessions over a period of 3 weeks or more.

The meta-analysis of imagined contact research suggests that greater elaboration of the imagined interaction can enhance its effectiveness (Miles \& Crisp, 2014). In particular, writing about the imagined interaction strengthens the effects of the intervention. For maximal effectiveness, participants need to mentally simulate an interaction, including conversations and feelings by imagining the positive traits of an outgroup member. Additionally, elaborating on the imagined interaction, such as in a writing task, can enhance the effect (Husnu \& Crisp, 2010). Imagined contact can be easily applied in education systems through storytelling or role-playing and can be used as the first step of prejudice intervention (Stathi \& Crisp, 2008).

Furthermore, perspective-taking, which refers to imagining the same scenario from different perspectives, has been explored. Prejudice-reduction effects were compared when participants imagined a positive interaction with an elderly person from a first-person perspective (through one's own eyes) or from a third-person perspective (through the eyes of an observer). The results suggested that, compared to a first-person perspective, a third-person perspective was more effective in producing the future intention of actual contact with the elderly (Crisp \& Husnu, 2011).

In extended and vicarious contact interventions, research also suggests that identification with the character who is engaging in intergroup contact augments the effects. For example, in the research with the Harry Potter books intervention, identification with the main character, Harry, resulted in more favourable views of the real-world stigmatized groups (Vezzali et al., 2015).

Thus, a number of studies have evaluated how to optimize indirect contact effects, particularly so that they can be used in interventions in applied settings. The future directions suggested below include other possibilities for optimizing interventions as well as relatively under-explored contexts for evaluating the effectiveness of indirect contact in promoting more favourable views of the other. 


\subsection{Future Directions}

Preliminary research suggests some promising avenues for future work on indirect contact. Some of this research has focused on how to optimize imagined contact for intervention in real-world settings to change reactions toward those who are different. One example comes from our research in Canada in which we investigated a variation of imagined contact that involved non-Asian youth (college and university students, aged approximately 18-22) imagining a close friend's friendly interaction with an East Asian person, imagined vicarious contact (Haji \& Noguchi, 2017). This was compared to a standard imagined contact condition (i.e. imagining one's own interaction with an East Asian person) and a neutral control condition (imagining an outdoor scene). We were interested in testing the effectiveness of imagined vicarious contact in promoting more favourable outgroup attitudes, because there has been a call for further research on how to optimize indirect contact interventions and because we believed that imagining a friend's positive interaction with an outgroup member may be less anxiety-provoking for some than imagining one's own interaction. Indeed, we found that imagined vicarious contact had a stronger prejudice-reducing effect on people with high Social Dominance Orientation (SDO; Pratto, Sidanius, Stallworth, \& Malle, 1994), i.e. a preference for their ingroup to have higher social status than other groups. Specifically, the result suggested that imagined contact can improve implicit attitudes of people high in SDO. Implicit attitudes are measured through the strength (speed) of associations with positive and negative words, rather than self-report questionnaires, and therefore can tap attitudes that people are unwilling to report (or unconscious attitudes that they are unaware they have). Those high in SDO may experience a change in their attitudes but may be unwilling to report this, possibly due to the desire to maintain their own group's social advantage. And if this is the case, imagined contact has particular relevance as a first step toward change in intergroup attitudes of those high in SDO who may be particularly resistant to change.

Other research investigating possible ways of optimizing imagined contact for intervention has used imagined I-sharing (Haji et al., 2017). In this research, we have investigated how imagining a shared experience (e.g. laughing at the same moment) with an outgroup member can be a particularly potent form of imagined contact. We have conducted studies with university students in Canada, the UK, and the Netherlands and have found that this variation of imagined contact has been particularly effective in promoting favourable attitudes toward a stigmatized outgroup (Muslims), at least among certain groups of people (e.g. those with no direct contact with the outgroup).

In addition to simply asking people about their attitudes and behaviour, as noted above, some research on indirect contact has assessed implicit attitudes - attitudes that people are unwilling to report due to concerns about portraying oneself in a positive light, or unable to report as they may be unaware that they have negative associations about certain groups. One widely used measure of implicit attitudes, the Implicit Associations Test (IAT; Greenwald, McGee, \& Schwartz, 1998), 
assesses reaction times to associations between groups and positive and negative words, in order to assess negative automatic associations with certain groups. Studies that have investigated the effects of imagined contact on implicit attitudes assessed with the IAT (e.g. Haji \& Noguchi, 2017; Turner \& Crisp, 2010) suggest that imagined contact can improve these automatic associations that people have with certain groups.

It has been noted that research on indirect contact effects on actual behaviour is limited (Miles \& Crisp, 2014). Similarly, most of the research on extended and vicarious contact described above looked at outcomes of attitudes and behaviour intentions. Therefore, the dearth of research evaluating actual behaviour seems to apply to indirect contact research more generally and suggests a very important avenue for future research.

Another limitation is that research to date has not typically investigated the lasting effects of indirect contact interventions beyond more than a few months (Lemmer \& Wagner, 2015). Although there is evidence of the lasting impact of indirect contact a month after end of intervention, we know little about whether effects last for a year or more (Lemmer \& Wagner, 2015).

Much of the research on indirect contact, particularly imagined contact, has involved laboratory research. Intervention research in educational settings is common for extended and vicarious contact, but interventions have often been conducted in multicultural societies that did not have a recent history of intergroup conflict or segregation. In a related vein, the review by Vezzali et al. (2014) noted that more research was needed on real-world interventions involving indirect contact in naturalistic settings. That being said, a meta-analysis of real-world indirect contact interventions (excluding imagined contact) has been conducted (Lemmer \& Wagner, 2015) and has found that these interventions do work in the real world, including in post-conflict settings.

Among the future directions that Vezzali et al. (2014) suggested was to consider how the type of relationship between the participant and the ingroup member in the indirect interaction influenced extended and vicarious conflict effects. They also suggested that it would be fruitful to compare the forms of indirect contact in terms of their relative effectiveness. Indeed, they suggested that different forms of indirect contact may reduce outgroup prejudice via different mechanisms (i.e. changing thoughts about the outgroup vs. decreasing anxiety about interacting with the outgroup). Furthermore, they called for additional research on indirect contact through media. As media-based interventions hold particular promise for practical application, this is an area where we look forward to seeing more research.

\subsection{Conclusion}

Changing prejudiced attitudes represents a step toward decreasing structural violence. And promoting more favourable views of the other among children and youth can help to impact structural violence now and in the future. Taken together, the 
studies described in this chapter suggest that there is considerable potential for indirect contact to improve intergroup attitudes among children and youth living in diverse societies. On the whole, interventions involving indirect contact are low cost and relatively easy to implement. In some cases, they have been shown to be particularly effective among children. Moreover, it is encouraging that research has not only found effects on attitudes, but also on intended behaviour and, through more rarely studied, actual behaviour toward those belonging to other groups. Indeed, it might be the case that younger generations' direct and indirect contact experiences influence adults' attitudes and behaviours, through the increase in children's crossgroup friendships and adults' consequent indirect and direct contact experiences with outgroup children, parents, and teachers. Indirect contact may increase not only the willingness for direct contact, but the likelihood of direct contact.

The first author, who grew up in the multicultural city of Toronto, Canada, recalls a high school social studies assignment in which she had an intercultural dinner exchange with another student in the class. She and the other student took turns going to each other's house for dinner to learn about each other's culture and traditions. The exchange was informative for the students and the parents and dispelled some misconceptions about the cultures. Whereas intercultural exchanges may be beneficial in promoting acceptance or friendship, the research described here suggests that even reading or watching videos about such intercultural exchanges have the potential to promote more positive views of the other. For children and youth living in settings that are less culturally diverse, infrequent opportunities for direct intergroup contact mean that there is actually greater potential for the effectiveness of indirect contact interventions. This highlights the potential power of indirect contact as a means for promoting peace in diverse and less diverse settings.

\section{References}

Allport, G. W. (1954). The nature of prejudice. Oxford, UK: Addison-Wesley Publishing Company. Birtel, M. D., \& Crisp, R. J. (2012). "Treating" prejudice an exposure-therapy approach to reducing negative reactions toward stigmatised groups. Psychological Science, 23(11), 1379-1386. https://doi.org/10.1177/0956797612443838

Cameron, L., \& Rutland, A. (2006). Extended contact through story reading in school: Reducing children's prejudice toward the disabled. Journal of Social Issues, 62(3), 469-488. https://doi. org/10.1111/j.1540-4560.2006.00469.x

Cameron, L., \& Rutland, A. (2007). Promoting children's positive intergroup attitudes towards stigmatized groups: Extended contact and multiple classification skills training. International Journal of Behavioural Development, 31(5), 454-466. https://doi. org/10.1177/0165025407081474

Cameron, L., Rutland, A., Brown, R., \& Douch, R. (2006). Changing children's intergroup attitudes toward refugees: Testing different models of extended contact. Child Development, 77(5), 1208-1219. https://doi.org/10.1111/j.1467-8624.2006.00929.x

Christie, D. J., Wagner, R. V., \& Winter, D. A. (Eds.). (2001). Peace, conflict, and violence: Peace psychology for the 21st century. Englewood Cliffs, NJ: Prentice-Hall.

Crisp, R. J., \& Husnu, S. (2011). Attributional processes underlying imagined contact effects. Group Processes \& Intergroup Relations, 14(2), 275-287. https://doi.org/10.1177/1368430210390721 
Crisp, R. J., Stathi, S., Turner, R. N., \& Husnu, S. (2009). Imagined intergroup contact: Theory, paradigm and practice. Social and Personality Psychology Compass, 3(1), 1-18. https://doi. org/10.1111/j.1751-9004.2008.00155.x

Gillig, T. K., \& Murphy, S. T. (2016). Fostering support for LGBTQ youth? The effects of a gay adolescent media portrayal on young viewers. International Journal of Communication, 10, 3838-3850. 1932-8036/20160005.

Greenwald, A. G., McGee, D. E., \& Schwartz, J. L. K. (1998). Measuring individual differences in implicit cognition: The implicit association test. Journal of Personality and Social Psychology, 74(6), 1464-1480. https://doi.org/10.1037/0022-3514.85.2.19710

Haji, R., \& Lalonde, R. N. (2017). If a close friend is from another religion, are you more open to other faiths? In M. Seedat, S. Suffla, \& D. Christie (Eds.), Enlarging the scope of peace psychology: African and world-regional contributions. New York, NY: Springer.

Haji, R., McKeown, S., \& Stathi, S. (2017, June). Turning a frown upside down: Imagining a shared laugh increases implicit and explicit liking. Paper presented at the 1st international symposium on intergroup communication, Thessaloniki, Greece.

Haji, R., \& Noguchi, H. (2017, May). Social dominance and cross-group friendship: Moderators of imagined contact on implicit views of an ethnic outgroup. Paper presented at the Biennial Symposium on the Contributions of Psychology to Peace, Rome \& Florence, Italy.

Husnu, S., \& Crisp, R. J. (2010). Elaboration enhances the imagined contact effect. Journal of Experimental Social Psychology, 46(6), 943-950. https://doi.org/10.1016/j.jesp.2010.05.014

Husnu, S., Mertan, B., \& Cicek, O. (2018). Reducing Turkish Cypriot children's prejudice toward Greek Cypriot: Vicarious and extended intergroup contact through storytelling. Group Processes \& Intergroup Relations, 21(1), 178-192. https://doi.org/10.1177/1368430216656469

Kang, S. K., \& Inzlicht, M. (2012). Stigma building blocks: How instruction and experience teach children about rejection by outgroups. Personality and Social Psychology Bulletin, 38, 357369. https://doi.org/10.1177/0146167211426729

Lemmer, G., \& Wagner, U. (2015). Can we really reduce ethnic prejudice outside the lab? A metaanalysis of direct and indirect contact interventions. European Journal of Social Psychology, 45, 152-168. https://doi.org/10.1002/ejsp.2079

Mazziotta, A., Mummendey, A., \& Wright, C. S. (2011). Vicarious intergroup contact effects: Applying social-cognitive theory to intergroup contact research. Group Processes and Intergroup Relations, 14(2), 255-274. https://doi.org/10.1177/1368430210390533

Miles, E., \& Crisp, R. J. (2014). A meta-analytic test of the imagined contact hypothesis. Group Processes \& Intergroup Relations, 17(1), 3-26. https://doi.org/10.1177/1368430213510573

Pettigrew, T. F. (2009). Secondary transfer effect of contact: Do intergroup contact effects spread to noncontacted outgroups? Social Psychology, 40, 55-65. https://doi.org/10.1027/ 1864-9335.40.2.55

Pettigrew, T. F., \& Tropp, L. R. (2006). A meta-analytic test of intergroup contact theory. Journal of Personality and Social Psychology, 90(5), 751-783. https://doi.org/10.1037/ 0022-3514.90.5.751

Pew Research Centre. (2018, May 29). Being Christian in Western Europe. Retrieved from: http:// www.pewforum.org/2018/05/29/nationalism-immigration-and-minorities/

Pratto, F., Sidanius, J., Stallworth, L. M., \& Malle, B. F. (1994). Social dominance orientation: A personality variable predicting social and political attitudes. Journal of Personality and Social Psychology, 67(4), 41-763. https://doi.org/10.1037/0022-3514.67.4.741

Stathi, S., \& Crisp, R. J. (2008). Imagining intergroup contact promotes projection to outgroups. Journal of Experimental Social Psychology, 44(4), 943-957. https://doi.org/10.1016/j. jesp.2008.02.003

Stathi, S., \& Crisp, R. J. (2009). Teaching and learning guide for: Imagined contact: Theory, paradigm, and practice. Social and Personality Psychology Compass, 3, 1129-1134. https://doi. org/10.1111/j.1751-9004.2009.00230.x

Stathi, S., Cameron, L., Hartley, B., \& Bradford, S. (2014). Imagined contact as a prejudicereduction intervention in schools: The underlying role of similarity and attitudes. Journal of Applied Social Psychology, 44, 536-546. https://doi.org/10.1111/jasp.12245 
Turner, R. N., \& Crisp, R. J. (2010). Imagining intergroup contact reduces implicit prejudice. British Journal of Social Psychology, 49(1), 129-142. https://doi.org/10.1348/0144666 09X419901

Turner, R. N., Crisp, R. J., \& Lambert, E. (2007). Imagining intergroup contact can improve intergroup attitudes. Group Processes and Intergroup Relations, 10(4), 427-441. https://doi. org/10.1177/1368430207081533

Turner, R. N., West, K., \& Christie, Z. (2013). Out- group trust, intergroup anxiety, and out-group attitude as mediators of the effect of imagined intergroup contact on intergroup behavioral tendencies. Journal of Applied Social Psychology, 43, E196-E205. https://doi.org/10.1111/ jasp. 12019

UNHCR. (2018). Figures at a Glance: Statistical Yearbooks. Retrieved from: http://www.unhcr. org/figures-at-a-glance.html

Vezzali, L., Hewstone, M., Capozza, D., Giovannini, D., \& Wöelfer, R. (2014). Improving intergroup relations with extended and vicarious forms of indirect contact. European Review of Social Psychology, 25, 314-389. https://doi.org/10.1080/10463283.2014.982948

Vezzali, L., Stathi, S., \& Giovannini, D. (2012). Indirect contact through book reading: Improving adolescents' attitudes and behavioural interventions toward immigrants. Psychology in the Schools, 49(2), 148-162. https://doi.org/10.1002/pits.20621

Vezzali, L., Stathi, S., Giovannini, D., Capozza, D., \& Trifiletti, E. (2015). The greatest magic of Harry Potter: Reducing prejudice. Journal of Applied Social Psychology, 45(2), 105-121. https://doi.org/10.1111/jasp.12279

Wright, S. C., Aron, A., McLaughlin-Volpe, T., \& Ropp, S. A. (1997). The extended contact effect: Knowledge of cross-group friendships and prejudice. Journal of Personality and Social Psychology, 73(1), 73-90. https://doi.org/10.1037/0022-3514.73.1.73

Open Access This chapter is licensed under the terms of the Creative Commons Attribution 4.0 International License (http://creativecommons.org/licenses/by/4.0/), which permits use, sharing, adaptation, distribution and reproduction in any medium or format, as long as you give appropriate credit to the original author(s) and the source, provide a link to the Creative Commons license and indicate if changes were made.

The images or other third party material in this chapter are included in the chapter's Creative Commons license, unless indicated otherwise in a credit line to the material. If material is not included in the chapter's Creative Commons license and your intended use is not permitted by statutory regulation or exceeds the permitted use, you will need to obtain permission directly from the copyright holder.

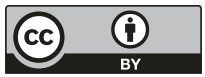

